\title{
Use of a 1.0 Tesla open scanner for evaluation of pediatric and congenital heart disease: a retrospective cohort study
}

Jimmy C Lu ${ }^{1,2^{*}}$, James C Nielsen ${ }^{3}$, Layne Morowitz ${ }^{3}$, Muzammil Musani ${ }^{4}$, Maryam Ghadimi Mahani ${ }^{2}$, Prachi P. Agarwal ${ }^{5}$, El-Sayed H. Ibrahim ${ }^{5}$ and Adam L. Dorfman ${ }^{1,2}$

\begin{abstract}
Background: Open cardiovascular magnetic resonance (CMR) scanners offer the potential for imaging patients with claustrophobia or large body size, but at a lower 1.0 Tesla magnetic field. This study aimed to evaluate the efficacy of open CMR for evaluation of pediatric and congenital heart disease.

Methods: This retrospective, cross-sectional study included all patients $\leq 18$ years old or with congenital heart disease who underwent CMR on an open 1.0 Tesla scanner at two centers from 2012-2014. Indications for CMR and clinical questions were extracted from the medical record. Studies were qualitatively graded for image quality and diagnostic utility. In a subset of 25 patients, signal-to-noise (SNR) and contrast-to-noise (CNR) ratios were compared to size- and diagnosis-matched patients with CMR on a 1.5 Tesla scanner.

Results: A total of 65 patients (median 17.3 years old, 60\% male) were included. Congenital heart disease was present in 32 (50\%), with tetralogy of Fallot and bicuspid aortic valve the most common diagnoses. Open CMR was used due to scheduling/equipment issues in 51 (80\%), claustrophobia in 7 (11\%), and patient size in $3(5 \%)$; 4 patients with claustrophobia had failed CMR on a different scanner, but completed the study on open CMR without sedation. All patients had good or excellent image quality on black blood, phase contrast, magnetic resonance angiography, and late gadolinium enhancement imaging. There was below average image quality in 3/63 (5\%) patients with cine images, and 4/15 (27\%) patients with coronary artery imaging. SNR and CNR were decreased in cine and magnetic resonance angiography images compared to 1.5 Tesla. The clinical question was answered adequately in all but 2 patients; 1 patient with a Fontan had artifact from an embolization coil limiting RV volume analysis, and in 1 patient the right coronary artery origin was not well seen.
\end{abstract}

Conclusions: Open 1.0 Tesla scanners can effectively evaluate pediatric and congenital heart disease, including patients with claustrophobia and larger body size. Despite minor artifacts and differences in SNR and CNR, the majority of clinical questions can be answered adequately, with some limitations with coronary artery imaging. Further evaluation is necessary to optimize protocols and image quality.

Keywords: Open scanner, Congenital heart disease, 1.0 Tesla

\footnotetext{
* Correspondence: jimmyl@umich.edu

'Department of Pediatrics and Communicable Diseases, Division of Pediatric

Cardiology, University of Michigan, Ann Arbor, MI, USA

${ }^{2}$ Department of Radiology, Section of Pediatric Radiology, University of

Michigan, Ann Arbor, MI, USA

Full list of author information is available at the end of the article
}

C Biomed Central (c) 2015 Lu et al.; licensee BioMed Central. This is an Open Access article distributed under the terms of the Creative Commons Attribution License (http://creativecommons.org/licenses/by/4.0), which permits unrestricted use, distribution, and reproduction in any medium, provided the original work is properly credited. The Creative Commons Public Domain Dedication waiver (http://creativecommons.org/publicdomain/zero/1.0/) applies to the data made available in this article, unless otherwise stated. 


\section{Background}

Cardiovascular magnetic resonance (CMR) is an indispensible tool for evaluation of cardiac anatomy and physiology in pediatric and congenital heart disease. CMR is particularly useful for adults with congenital heart disease, in whom acoustic windows often limit the utility of echocardiography $[1,2]$. However, this population may be at increased risk for obesity $[3,4]$, which can limit use of CMR. Short bore 1.5 Tesla scanners, the primary tool in this population, or 3.0 Tesla scanners can be limited by patient size and difficulty with claustrophobia, as patients must lie still within a circumferential bore. "Open" scanners allow more room to the sides of the patient, and may be more tolerable for patients with claustrophobia or larger body size [5]. At a lower magnetic field of 1.0 Tesla, there are also theoretical advantages, e.g. lower specific absorption rate (SAR) and less B1 shading artifact. However, there is a paucity of data regarding application of 1.0 Tesla scanners in pediatric and congenital heart disease [6,7], and it is unclear whether the lower magnetic field and signal to noise ratio is adequate to evaluate pediatric patients with smaller body size, or adult congenital heart disease patients, with more complicated cardiac anatomy. This retrospective study aimed to evaluate the efficacy of open scanners in this population.

\section{Methods}

\section{Patient selection}

This multicenter retrospective cohort study included all patients who underwent CMR for evaluation of pediatric or congenital heart disease on an open scanner at Stony Brook University or the University of Michigan C.S. Mott Children's Hospital, between 2/8/2012 and 7/24/2014. This study was approved by the Institutional Review Boards at both centers, and requirement for informed consent in this retrospective study was waived. Patients of any age with congenital heart disease were included; patients without congenital heart disease were included if they were 18 years or younger at the time of CMR. Patients were excluded if digital images were not available for review. Medical records were reviewed for patient demographics, indication for the study, and reason for performing the study on the open scanner.

For quantitative comparison of signal-to-noise ratio (SNR) and contrast-to-noise ratio (CNR), a subset of 25 CMR studies were matched 1:1 by patient body surface area and diagnosis with CMR studies performed on a 1.5 Tesla scanner at the same institution. It should be noted that SNR and CNR depend on a number of imaging parameters, including voxel size, acceleration factor, readout bandwidth, and number of averages. Therefore, imaging parameters on both scanners are summarized in Table 1.

\section{CMR imaging}

CMR was performed with a Panorama High Field Open 1.0 Tesla scanner (Philips, Best, The Netherlands), using a solenoid body coil (medium, large or extra large as appropriate for patient size). Gradient amplitude is $28 \mathrm{mT} / \mathrm{m}$; maximum slew rate is $120 \mathrm{mT} / \mathrm{m} / \mathrm{ms}$. Sensitivity encoding (SENSE) was not used for image acquisition, i.e. SENSE acceleration factor $=1$, to maintain adequate signal-tonoise ratio (SNR). Typical imaging parameters are presented in Table 1, with some minor differences by center. Cine imaging was performed with a breathhold, electrocardiographic gated, segmented k-space balanced steadystate with free precession (SSFP) sequence. Black blood imaging was performed with a breathhold, electrocardiographic gated, double inversion recovery turbo spin echo sequence. Imaging parameters varied by weighting of the sequence. Phase contrast imaging was performed free-breathing. Magnetic resonance angiogram (MRA) was performed with a T1-weighted fast field echo sequence, after injection of $0.2 \mathrm{mmol} / \mathrm{kg}$ of gadoteridol (ProHance, Bracco, Monroe Township, New Jersey) for studies performed at the University of Michigan, or gadopentetate dimeglumine (Magnevist, Bayer, Leverkusen, Germany) for studies performed at Stony Brook University. Two post-contrast dynamics were performed to highlight the anatomy of interest (e.g. pulmonary arteries or aorta) as well as the venous phase. Coronary artery imaging was performed with a respiratory navigator gated, electrocardiographic gated, three-dimensional SSFP sequence. Late gadolinium enhancement (LGE) was performed 12-15 minutes after contrast injection, using a breathhold, electrocardiographic gated, phase-sensitive inversion recovery sequence. In 12 patients at the University of Michigan, LGE imaging was performed with a three-dimensional, respiratory navigator gated, electrocardiographic gated, phase-sensitive inversion recovery sequence, to improve in-plane spatial resolution while maintaining adequate SNR.

CMR at 1.5 Tesla was performed on a commercially available scanner (Ingenia, Philips, Best, The Netherlands), using a phased-array body surface coil. Gradient amplitude is $33 \mathrm{mT} / \mathrm{m}$; maximum slew rate is $200 \mathrm{mT} / \mathrm{m} / \mathrm{ms}$. Typical imaging parameters are presented in Table 1. It should be noted that TR was longer on the 1.0 Tesla scanner than the 1.5 Tesla scanner due to the lower gradient performance characteristics (maximum gradient strength and slew rate) of the 1.0 Tesla scanner. Phase contrast imaging was performed free-breathing. MRA was performed with two post-contrast dynamics, which was sufficient to highlight the anatomy of interest, e.g. pulmonary arteries or aorta, as well as the venous phase of the contrast uptake. LGE was performed using a breathhold, electrocardiographic gated, phase-sensitive inversion recovery sequence. 
Table 1 Typical imaging parameters at 1.0 Tesla and 1.5 Tesla

\begin{tabular}{|c|c|c|}
\hline & 1.0 Tesla & 1.5 Tesla \\
\hline \multicolumn{3}{|l|}{ Cine SSFP } \\
\hline $\mathrm{TR}(\mathrm{ms}) / \mathrm{TE}(\mathrm{ms})$ & $4.1-4.4 / 2.0-2.2$ & $3.1-3.3 / 1.6$ \\
\hline Flip angle $\left(^{\circ}\right)$ & $55-60$ & 60 \\
\hline Receive bandwidth (Hz/pixel) & 578 or 1014 & 954 \\
\hline In-plane spatial resolution (mm) & $1.9-2.0$ & $1.7-1.9$ \\
\hline Slice thickness (mm) & 8 & 8 \\
\hline Phases per cardiac cycle & $24-30$ & 30 \\
\hline SENSE & 1 & 2 \\
\hline$S_{A R}^{*}$ & $<74-92 \%$ & $<95 \%$ \\
\hline \multicolumn{3}{|l|}{ Black blood } \\
\hline In-plane spatial resolution (mm) & $1.6-1.7$ & $1.2-1.5$ \\
\hline Slice thickness (mm) & $5-7$ & $4-5$ \\
\hline Number of signals averaged & $1-2$ & 1 \\
\hline SENSE & 1 & 2 \\
\hline SAR (for proton density) & $<34 \%$ & $<15 \%$ \\
\hline \multicolumn{3}{|l|}{ Phase contrast } \\
\hline $\mathrm{TR}(\mathrm{ms}) / \mathrm{TE}(\mathrm{ms})$ & $6.5-6.9 / 4.1-4.7$ & $3.1-3.3 / 1.6$ \\
\hline Flip angle $\left(^{\circ}\right)$ & $12-25$ & 12 \\
\hline Receive bandwidth (Hz/pixel) & $318-364$ & 717 \\
\hline In-plane spatial resolution (mm) & $2.0-2.2$ & $1.6-1.8$ \\
\hline Slice thickness (mm) & 6 & 6 \\
\hline Phases per cardiac cycle & $20-40$ & 40 \\
\hline Number of signals averaged & $2-3$ & 3 \\
\hline SENSE & 1 & 2 \\
\hline SAR & $<2 \%$ & $<2 \%$ \\
\hline \multicolumn{3}{|l|}{ Magnetic resonance angiogram } \\
\hline $\mathrm{TR}(\mathrm{ms}) / \mathrm{TE}(\mathrm{ms})$ & $4.0 / 1.5$ & $4.8 / 1.5$ \\
\hline Flip angle $\left(^{\circ}\right)$ & 40 & 40 \\
\hline Receive bandwidth (Hz/pixel) & 482 or 789 & 249 \\
\hline In-plane spatial resolution (mm) & $1.8-2.2$ & $1.7-1.8$ \\
\hline Slice thickness (mm) & $3.0-3.4$ & $2.6-2.8$ \\
\hline SENSE & 1 & $1.5 \mathrm{AP}, 1.5 \mathrm{LR}$ \\
\hline SAR & $<82-92 \%$ & $<51 \%$ \\
\hline \multicolumn{3}{|l|}{ 3-dimensional SSFP } \\
\hline $\mathrm{TR}(\mathrm{ms}) / \mathrm{TE}(\mathrm{ms})$ & $5.1-5.5 / 2.6-2.8$ & $4.3 / 2.2$ \\
\hline Flip angle $\left(^{\circ}\right)$ & 90 & 90 \\
\hline Receive bandwidth (Hz/pixel) & 578 & 618 \\
\hline Isotropic voxel size (mm) & $1.9-2.6$ & 1.6 \\
\hline SENSE & 1 & $1.5 \mathrm{AP}, 1.5 \mathrm{LR}$ \\
\hline SAR & $<62 \%$ & $<36 \%$ \\
\hline
\end{tabular}

Table 1 Typical imaging parameters at 1.0 Tesla and 1.5 Tesla (Continued)

LGE

\begin{tabular}{|c|c|c|}
\hline $\mathrm{TR}(\mathrm{ms})$ & 6.0 & 6.1 \\
\hline $\mathrm{TE}(\mathrm{ms})$ & 3.0 & 3.0 \\
\hline Flip angle $\left(^{\circ}\right)$ & 25 & 25 \\
\hline Receive bandwidth (Hz/pixel) & 299 or 495 & 232 \\
\hline In-plane spatial resolution (mm) & $2.0-2.2$ & $1.7-1.8$ \\
\hline Slice thickness (mm) & 8 & 8 \\
\hline SENSE & 1 & 1 \\
\hline SAR & $<10 \%$ & $<8 \%$ \\
\hline \multicolumn{3}{|l|}{ 3-dimensional LGE } \\
\hline $\mathrm{TR}(\mathrm{ms}) / \mathrm{TE}(\mathrm{ms})$ & $5.3 / 2.6$ & Not performed \\
\hline Flip angle $\left(^{\circ}\right)$ & 25 & \\
\hline Receive bandwidth (Hz/pixel) & 299 & \\
\hline In-plane spatial resolution (mm) & 1.9 & \\
\hline Slice thickness (mm) & 5 & \\
\hline SAR & $<17 \%$ & \\
\hline
\end{tabular}

Scan time for both scanners was measured from the start of the first imaging sequence through the final imaging sequence. Thus, this included patient rest time between breath-holds, and potential need to pause during imaging if there was any patient discomfort.

\section{Image analysis}

All images were evaluated by a single experienced observer. For each sequence type, image quality was scored qualitatively, taking into consideration resolution, blurring effects, low SNR, delineation of structures, and artifacts, according to a 4-point scale (4- excellent with no artifacts; 3 - good with minor artifacts; 2 - below average, with significant artifacts affecting interpretation; 1 - poor, nondiagnostic) [8]. Imaging examples are presented in Fig. 1.

Clinical questions were extracted from the medical record, and were categorized as: ventricular size/function, pulmonary artery anatomy, regurgitant fraction, LGE, aortic root dimensions, coronary artery anatomy, aortic arch anatomy, ratio of pulmonary to systemic blood flow (Qp:Qs ratio), and pulmonary venous anatomy. A given study could have multiple clinical questions. Studies were scored for diagnostic utility on a 4-point scale, based on the interpreter's confidence in their ability to answer the specific clinical questions ( 4 - high confidence of diagnosis; 3 - answers question adequately; 2 - low confidence; 1 - could not answer the clinical question). Ventricular 


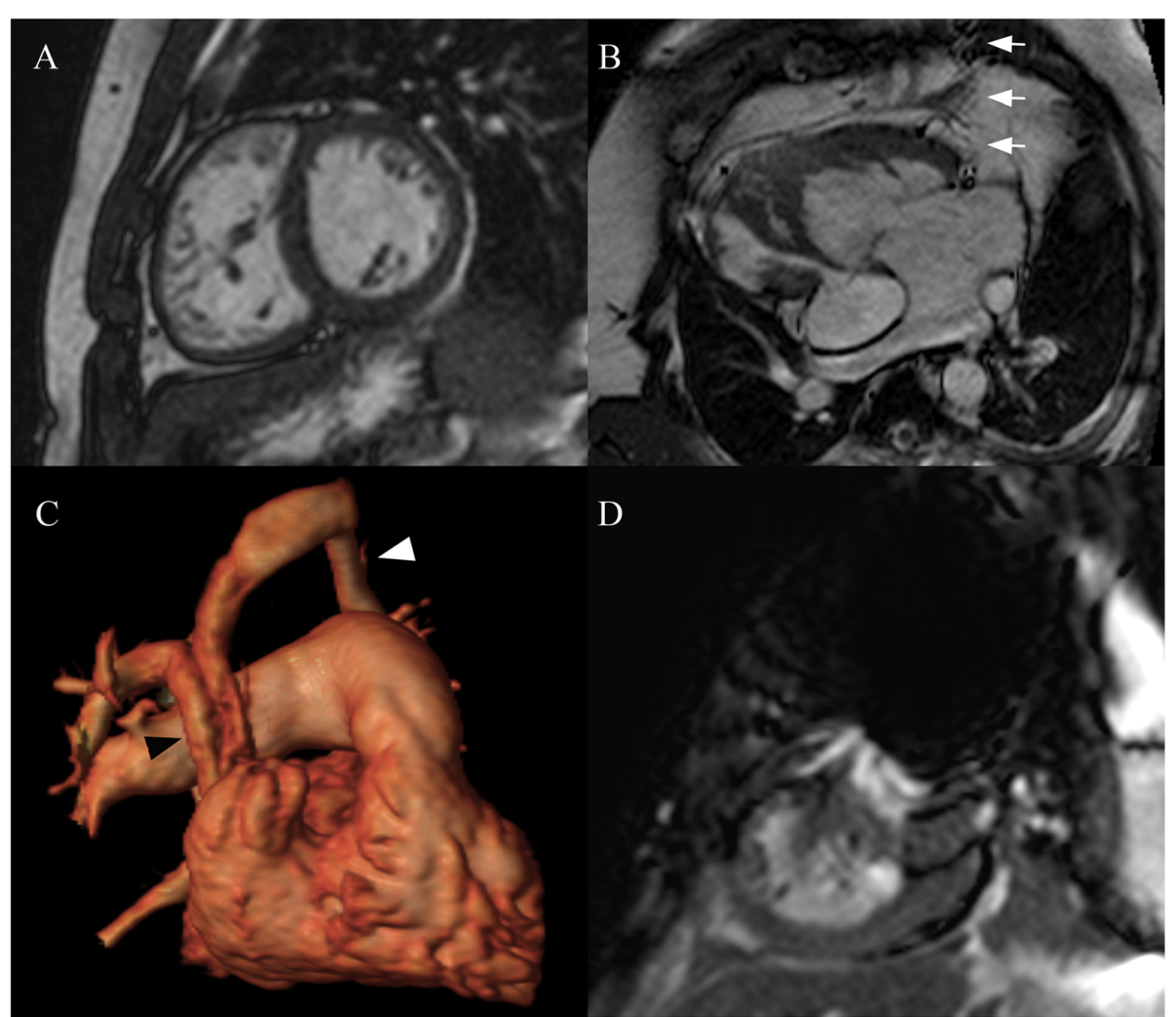

Fig. 1 Images were scored on a 4-point scale for image quality and diagnostic utility. (a) SSFP imaging in short-axis in a 26 year-old patient with tetralogy of Fallot and complete atrioventricular septal defect status post repair: No artifacts and good endocardial definition, scored 4 for both quality and diagnostic utility. (b) SSFP imaging in the four-chamber plane in a 32 year-old patient with dextrocardia and congenitally corrected transposition of the great arteries, who previously failed CMR on a 1.5 Tesla scanner: Flow-related artifact (arrows), which did not affect interpretation of ventricular size or function, scored 3 for quality, but 4 for diagnostic utility. (c) Volume-rendered reconstruction of gadolinium-enhanced MRA in a 57 year-old patient with pulmonary hypertension and reported history of atrial septal defect repair in a foreign country. The left upper pulmonary vein (white arrowhead) drains into the left innominate vein; the right upper and middle veins drain into a baffle within the superior vena cava (black arrowhead) to the left atrium. Scored 4 for both quality and diagnostic utility. (d) SSFP imaging in short axis in a 16 year-old patient with unbalanced atrioventricular septal defect status post Fontan: Significant coil artifact, obscuring portions of the heart, scored 2 for both quality and diagnostic utility (for right ventricular size and function)

size/function was scored on the ability to define and contour the endocardial border. Anatomy of the pulmonary arteries, coronary arteries, aortic arch, and pulmonary veins was scored on definition of the respective structures, course, and presence/absence of stenosis as appropriate. Flow measurements were scored by internal consistency of data. LGE images were scored by ability to determine presence or absence of LGE, and extent if present.

Due to the qualitative nature of grading image quality and diagnostic utility, a $20 \%$ subset of the cohort was randomly selected to evaluate reproducibility. A second reader, blinded to the scores of the first reader, reevaluated the images to evaluate interobserver agreement. The initial reader, blinded to initial scores, also reevaluated the images to evaluate intraobserver agreement.

SNR and CNR were calculated for cine SSFP, black blood, MRA, 3-dimensional SSFP, and LGE images. A region of interest (ROI) of approximately $1.0 \mathrm{~cm}^{2}$ was drawn in two locations (Fig. 2). For cine SSFP and LGE images, a midventricular short-axis slice was used, and ROIs were drawn in the blood pool and in the interventricular septum. For black blood and 3-dimensional SSFP images, ROIs were drawn in the blood pool and in any adjacent myocardium. For the MRA, the first postcontrast dynamic was used, and ROIs were drawn in the aorta or main pulmonary artery (depending on timing of the contrast) and in the lung field. SNR was defined as the mean signal intensity in the blood pool (for cine SSFP, MRA, 3-dimensional SSFP and LGE images) or myocardium (for black blood images) divided by the standard deviation of signal intensity in that ROI [9]. CNR was defined as the difference in signal intensities of the two ROIs, divided by the average of the standard deviations of the two ROIs [9]. 


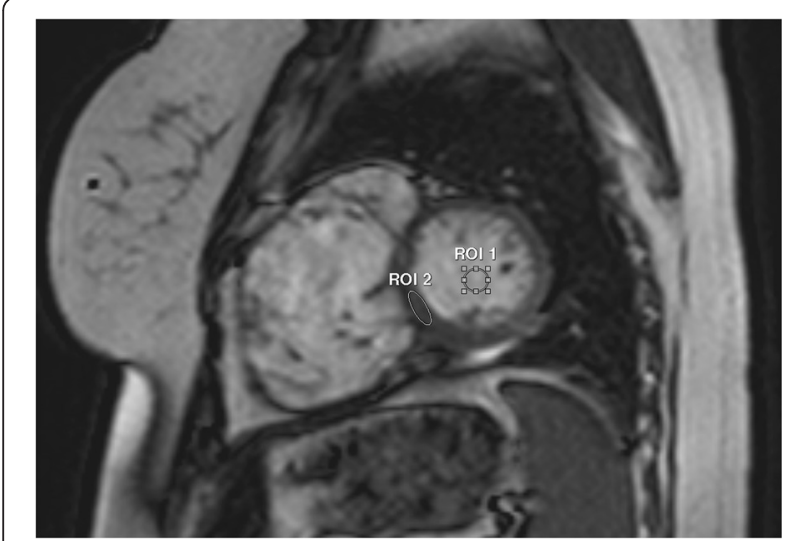

Fig. 2 Regions of interest for contrast to noise measurement. For cine images, an $\mathrm{ROI}$ of $\sim 1.0 \mathrm{~cm}^{2}$ was drawn in the blood pool and in the septum. Only ROI1 (the blood pool) was used for signal to noise ratio measurement

\section{Statistical analysis}

Data are presented as median (interquartile range [IQR]), mean \pm standard deviation, or number (percent), as appropriate. SNR, CNR, and scan time on 1.0 Tesla and 1.5 Tesla studies were compared for each sequence using t-test. Age and body surface area among patients on 1.0 Tesla and 1.5 Tesla patients were not normally distributed, and were compared using Wilcoxon matched-pairs signed rank test. A p-value $<0.05$ was considered statistically significant. Intraobserver and interobserver agreement was calculated using weighted kappa, to take into consideration closeness of agreement.

\section{Results}

A total of 65 patients were included, with demographic data presented in Table 2. The majority of the patients were adolescents and adults, with 6 patients under the age of 10 , and the youngest 3.5 years old. Patients were referred to the open scanner primarily due to scheduling reasons, although 10 (15\%) were referred for claustrophobia or patient size. Among patients referred for claustrophobia or body size, 4 had failed a previous attempt on a 1.5 Tesla scanner; all 4 patients successfully completed the study on the open scanner, without sedation. Of the remaining 6 patients, 4 were referred for first CMR on the open scanner; 2 had previously had a successful scan on a standard scanner. Only 2 patients in this cohort required sedation, due to age or developmental delay. Apart from sternal wires (20/65 patients, $31 \%)$, implanted metal objects were rare (1 with an embolization coil, 1 with a pulmonary artery stent, 1 with Harrington rods). Mean scan time was $54.1 \pm 22.4$ minutes. In the matched cohort of 1.0 Tesla and 1.5 Tesla patients, there was no significant difference in scan time $(58.9 \pm 20.9$ vs. $51.7 \pm 12.7 \mathrm{~min}, \mathrm{p}=0.15)$.
Table 2 Demographic data $(N=65)$

\begin{tabular}{|c|c|}
\hline Age (years) & $17.3(14.7-20.9)$ \\
\hline Male gender & $39(60 \%)$ \\
\hline Height (cm) & $166(154-175)$ \\
\hline Weight (kg) & $71.0(60.5-87.7)$ \\
\hline Body surface area $\left(\mathrm{m}^{2}\right)$ & $1.83(1.67-2.04)$ \\
\hline Diagnosis of congenital heart disease & $33(51 \%)$ \\
\hline Tetralogy of Fallot s/p repair & 8 \\
\hline Bicuspid aortic valve & 6 \\
\hline Aortic stenosis & 4 \\
\hline Coarctation s/p repair & 2 \\
\hline Ebstein anomaly & 2 \\
\hline Partial anomalous pulmonary venous connections & 2 \\
\hline Pulmonary stenosis & 2 \\
\hline Other & 7 \\
\hline \multicolumn{2}{|l|}{ Indications for CMR } \\
\hline Left ventricular size/function & $53(82 \%)$ \\
\hline Right ventricular size/function & $26(40 \%)$ \\
\hline Regurgitant fraction & $23(35 \%)$ \\
\hline Pulmonary artery anatomy & $16(25 \%)$ \\
\hline Aortic root dimensions & $15(23 \%)$ \\
\hline Late gadolinium enhancement & $15(23 \%)$ \\
\hline Coronary artery anatomy & $12(18 \%)$ \\
\hline Aortic arch anatomy & $10(15 \%)$ \\
\hline Qp:Qs ratio & $7(11 \%)$ \\
\hline Pulmonary venous anatomy & $4(6 \%)$ \\
\hline \multicolumn{2}{|l|}{ Reason for open scanner } \\
\hline Scheduling/equipment issue & $52(80 \%)$ \\
\hline Claustrophobia & $7(11 \%)$ \\
\hline Patient size & $3(5 \%)$ \\
\hline Unspecified & $3(5 \%)$ \\
\hline
\end{tabular}

Data given as median (IQR) or number (percent). Qp:Qs, ratio of pulmonary to systemic blood flow

\section{Image quality}

All patients had good or excellent image quality on black blood, phase contrast, MRA, and LGE imaging (Fig. 3). There was below average image quality in $3 / 63$ (5\%) patients with cine images, and 4/15 (27\%) patients with coronary artery imaging; all other sequences had at least good image quality in all patients. No sequences were rated as poor/nondiagnostic. Intraobserver agreement for grading image quality was good, with weighted kappa of 0.73. Interobserver agreement was fair, with weighted kappa of 0.32 . The majority of disagreement was in differentiating between good and excellent image quality; the raters had $85 \%$ agreement in differentiating image quality 2 from values of 3-4. 


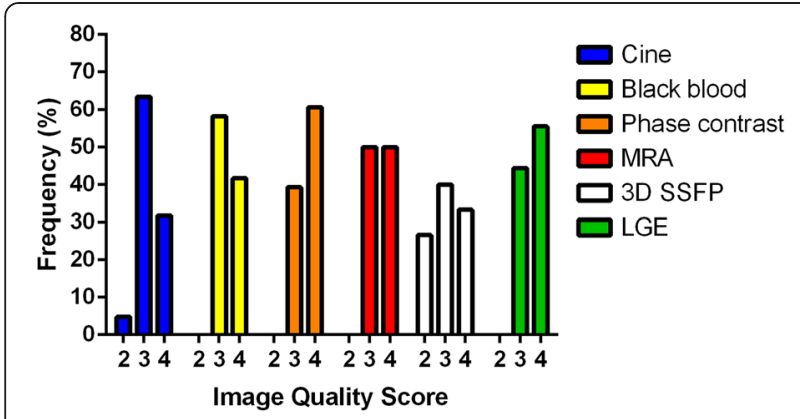

Fig. 3 Histogram of image quality score, by imaging sequence. MRA, magnetic resonance angiogram. 3D SSFP, three-dimensional steady state free precession. LGE, late gadolinium enhancement

In the subset of 25 patients for SNR and CNR analysis, there was no difference between patients on the 1.0 Tesla and 1.5 Tesla scanners in age (median 20.6, IQR 15.4-34.9, vs. 22.4, IQR $16.0-22.2, \mathrm{p}=0.60)$ or body surface area (1.83, IQR 1.62-2.09, vs. 1.84, IQR 1.66-2.07, $\mathrm{p}=0.62$ ). Both SNR (Fig. 4) and CNR (Fig. 5) were decreased on the open scanner for cine SSFP and MRA images, but similar to the 1.5 Tesla scanner for black blood, 3-dimensional SSFP, and LGE images. Use of 3-dimensional LGE imaging markedly increased SNR $(26.5 \pm 12.3$ vs. $9.0 \pm 0.8, \mathrm{p}=0.004)$ and CNR $(19.2 \pm 5.9$ vs. $7.0 \pm 1.4, \mathrm{p}=0.0003$ ) compared to 2-dimensional LGE imaging at 1.0 Tesla.

\section{Diagnostic utility}

The clinical question was answered adequately (score $3+$ ) in all but 2 patients (Fig. 6). In 1 patient status post Fontan procedure, susceptibility artifact due to an embolization coil limited evaluation of right ventricular volume. In 1 patient, the right coronary artery course appeared normal,

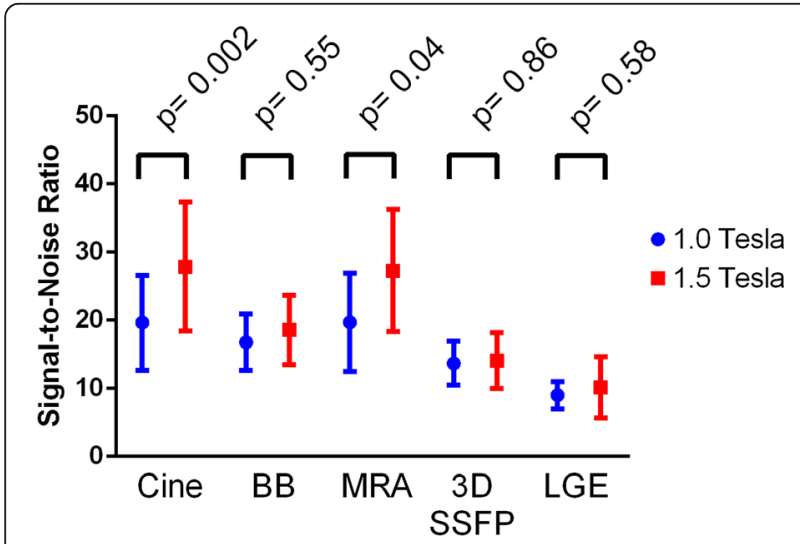

Fig. 4 Signal-to-noise ratio by sequence on 1.0 Tesla open or standard 1.5 Tesla scanner. BB, black blood; MRA, magnetic resonance angiogram; 3D SSFP, 3-dimensional steady state free precession; LGE, late gadolinium enhancement

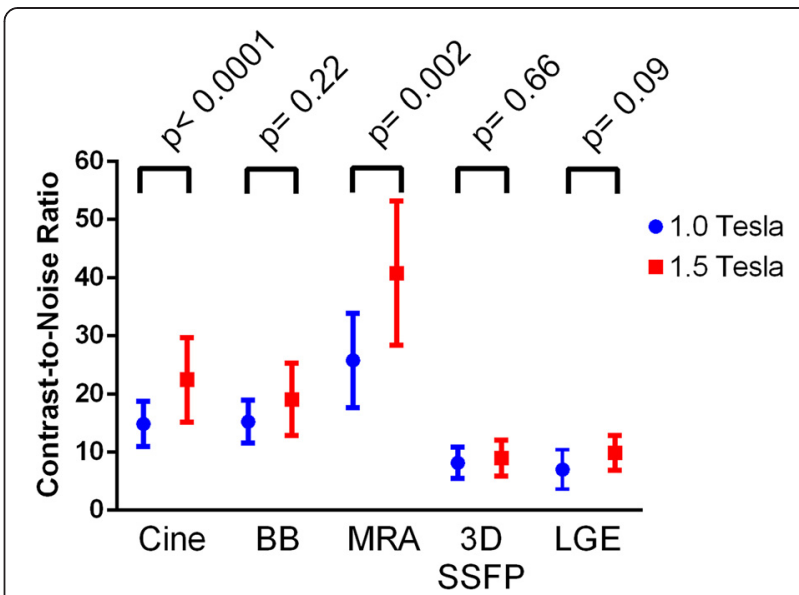

Fig. 5 Contrast-to-noise ratio by sequence on 1.0 Tesla open or standard 1.5 Tesla scanner. BB, black blood; MRA, magnetic resonance angiogram; 3D SSFP, 3-dimensional steady state free precession; LGE, late gadolinium enhancement

although the origin was suboptimally visualized; the left coronary artery was normal. No studies were scored as nondiagnostic, and no patients required callback for reevaluation on a different scanner. Intraobserver and interobserver agreement for grading diagnostic utility was good, with weighted kappa 0.85 and 0.61 , respectively. Again, the vast majority of disagreement was differentiating whether the clinical question was answered adequately or confidently; there was $97 \%$ agreement in differentiating score 2 from score 3-4.

\section{Discussion}

Open 1.0 Tesla scanners can be effective for evaluation of selected groups of patients with pediatric and congenital heart disease. Despite minor artifacts and decreased SNR and CNR, the majority of clinical questions can be answered adequately. To our knowledge, this is the first systematic report of CMR at 1.0 Tesla in the pediatric and congenital heart disease population.

The primary advantage of open scanners is the ability to increase the population eligible for CMR, including patients with larger body size or claustrophobia. A prior study demonstrated decreased anxiety in claustrophobic patients undergoing open CMR [5], although a randomized controlled trial demonstrated a significant persistent rate of claustrophobic events in the open scanner [10]. These studies included primarily patients with magnetic resonance imaging of other body parts; patients undergoing CMR, who must have the torso within the bore, could potentially be at higher risk for failure of CMR in a closed bore scanner. Although limited to a small subset of this cohort, the current results are promising, as all 4 patients who had failed a prior scan on a closed bore scanner completed the study on the open 


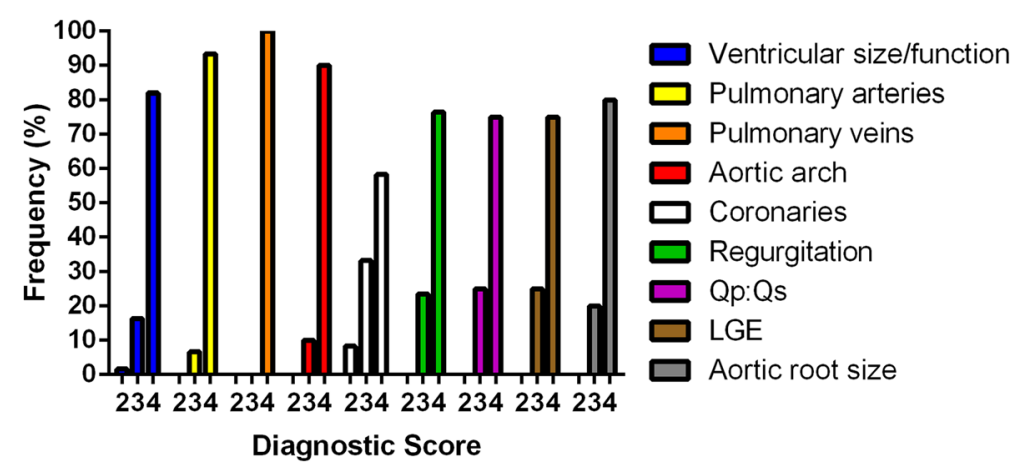

Fig. 6 Histogram of diagnostic utility, by clinical question. Qp:Qs, ratio of pulmonary to systemic blood flow. LGE, late gadolinium enhancement

scanner without sedation. Prospective identification of patients who may benefit from the open scanner could prevent lost scanner time or need to reschedule.

In this cohort, the majority of patients were referred to the open scanner due to scheduling or equipment issues, suggesting the primary practical advantage may be availability of scanner time. However, the diagnostic success of open CMR studies in this cohort confirms that selected patients in this population can appropriately take advantage of this scanner. Further work is needed to identify additional populations that can appropriately take advantage of open scanner availability.

Due to the lower magnetic field, signal to noise ratio suffers, and modifications to existing protocols are necessary to optimize image quality. De Bucourt and colleagues reported decreased SNR and CNR in extremely obese patients with an open 1.0 Tesla scanner [11]. However, this study used the integrated body coil, rather than the standard body coil, which was used for regular weight control patients. All patients in the current study used the standard body coil, which may partially explain more similar SNR and CNR in some sequences to studies at 1.5 Tesla. Furthermore, techniques to maximize signal, such as increased voxel size, avoidance of parallel imaging [12], and multiple signal averaging may partially offset differences due to magnetic field strength, at the cost of scan time and spatial resolution. In this cohort, studies on the 1.0 Tesla scanner had lower spatial resolution with more frequent usage of multiple signal averaging, without parallel imaging. By adjusting these parameters, SNR and CNR were actually similar among 1.0 Tesla and 1.5 Tesla scanners for black blood, 3-dimensional SSFP, and LGE imaging, but remained decreased for cine SSFP images and MRA. SNR and CNR could be further improved for LGE imaging with a 3-dimensional sequence, which is consistent with prior data at 1.5 Tesla [13]. Further evaluation is necessary for optimization of image quality, to minimize potential for diagnostic error. Furthermore, more technical and quantitative analysis may be necessary particularly for phase contrast data. Longer echo time has been shown to increase risk for error in flow measurements, due to intravoxel dephasing, particularly with stenotic jets [14-16]. This retrospective study did not allow for direct comparison of flow measurements in the same patients at 1.0 Tesla and 1.5 Tesla.

Despite some differences in image quality, the diagnostic score best reflects the clinical potential of the open scanner. Pertinent clinical findings were still detectable, particularly when the focus was ventricular size and systolic function. However, further optimization remains necessary, particularly for coronary artery imaging, which was the most limited of the indications in this study, or when evaluating small vessels. This cohort included few small children; further study is necessary, but patient selection based on clinical indication may be necessary.

This study has several limitations. Retrospective chart review is limited by documentation for determining reasons for the study or reasons for using the open scanner. However, only 3 patients did not have an identified reason for the open scanner. The retrospective nature of this study also prevented standardization of the imaging protocols, particularly between centers; further prospective study is necessary to optimize imaging parameters at 1.0 Tesla. This cohort consisted primarily of adolescents and adults, which may reflect selection bias. Although extrapolation to younger patients may not be appropriate, this does highlight that this population may benefit from use of the open scanner. This cohort cannot address whether need for sedation in smaller children can be lessened with the open scanner. The small sample size limits power to detect small differences in SNR and CNR, and verification in a larger cohort is necessary.

\section{Conclusions}

Open 1.0 Tesla scanners can successfully evaluate pediatric and congenital heart disease in selected patients. Despite some differences in image quality, SNR and $\mathrm{CNR}$, the vast majority of studies were adequate to 
answer the clinical questions. Further work is necessary to optimize protocols for these indications, and to better define patient characteristics appropriate to this scanner. Use of the open scanner in this population could increase the potential cohort who can tolerate CMR without sedation or discomfort.

\section{Competing interests}

The authors declare that they have no competing interests.

\section{Authors' contributions}

$J C L$ conceived of the study, participated in its design and coordination, analyzed the images, performed the statistical analysis, and helped to draft the manuscript. JCN participated in study design and coordination. LM and MM extracted demographic data and clinical indications. MGM and PPA assisted with study design and data collection. El-SHI assisted in interpretation of the data. ALD conceived of the study, participated in its design, and helped to draft the manuscript. All authors critically reviewed and approved the final manuscript.

\section{Acknowledgements}

This work had no external funding.

\section{Author details}

${ }^{1}$ Department of Pediatrics and Communicable Diseases, Division of Pediatric Cardiology, University of Michigan, Ann Arbor, MI, USA. ²Department of Radiology, Section of Pediatric Radiology, University of Michigan, Ann Arbor MI, USA. 'Departments of Pediatrics and Radiology, Stony Brook University, Stony Brook, NY, USA. ${ }^{4}$ Department of Medicine, Division of Cardiology, Stony Brook University, Stony Brook, NY, USA. 'Department of Radiology, Division of Cardiothoracic Radiology, University of Michigan, Ann Arbor, MI, USA.

Received: 21 November 2014 Accepted: 4 May 2015

Published online: 25 May 2015

\section{References}

1. Valente AM, Cook S, Festa P, Ko HH, Krishnamurthy R, Taylor AM, et al. Multimodality imaging guidelines for patients with repaired tetralogy of fallot: a report from the American society of echocardiography: developed in collaboration with the society for cardiovascular magnetic resonance and the society for pediatric radiology. J Am Soc Echocardiogr. 2014;27:111-41.

2. Warnes CA, Williams RG, Bashore TM, Child JS, Connolly HM, Dearani JA, et al. ACC/AHA 2008 guidelines for the management of adults with congenital heart disease: a report of the American college of cardiology/ American heart association task force on practice guidelines (writing committee to develop guidelines on the management of adults with congenital heart disease). Circulation. 2008;118:e714-833.

3. Pemberton VL, McCrindle BW, Barkin S, Daniels SR, Barlow SE, Binns HJ, et al. Report of the national heart, lung, and blood Institute's working group on obesity and other cardiovascular risk factors in congenital heart disease. Circulation. 2010;121:1153-9.

4. Smith-Parrish M, Yu S, Rocchini A. Obesity and elevated blood pressure following repair of coarctation of the aorta. J Pediatr. 2014;164:1074-8.

5. Bangard C, Paszek J, Berg F, Eyl G, Kessler J, Lackner K, et al. MR imaging of claustrophobic patients in an open 1.0T scanner: motion artifacts and patient acceptability compared with closed bore magnets. Eur J Radiol. 2007;64:152-7.

6. Rupprecht T, Nitz W, Wagner M, Kreissler P, Rascher W, Hofbeck M. Determination of the pressure gradient in children with coarctation of the aorta by low-field magnetic resonance imaging. Pediatr Cardiol. 2002;23:127-31.

7. Tzifa A, Komnou A, Loggitsi D. Cardiac magnetic resonance imaging in a premature baby with interrupted aortic arch and aortopulmonary window. Cardiol Young. 2013;23:742-5.

8. Naehle CP, Kreuz J, Strach K, Schwab JO, Pingel S, Luechinger R, et al. Safety, feasibility, and diagnostic value of cardiac magnetic resonance imaging in patients with cardiac pacemakers and implantable cardioverters/defibrillators at 1.5 T. Am Heart J. 2011;161:1096-105.
9. Groarke JD, Waller AH, Vita TS, Michaud GF, Di Carli MF, Blankstein R, et al. Feasibility study of electrocardiographic and respiratory gated, gadolinium enhanced magnetic resonance angiography of pulmonary veins and the impact of heart rate and rhythm on study quality. J Cardiovasc Magn Reson. 2014;16:43.

10. Enders J, Zimmermann E, Rief M, Martus P, Klingebiel R, Asbach $P$, et al. Reduction of claustrophobia with short-bore versus open magnetic resonance imaging: a randomized controlled trial. PLoS One. 2011;6:e23494.

11. De Bucourt M, Streitparth F, Wonneberger U, Rump J, Teichgraber U. Obese patients in an open MRI at 1.0 Tesla: image quality, diagnostic impact and feasibility. Eur Radiol. 2011;21:1004-15.

12. Biglands JD, Radjenovic A, Ridgway JP. Cardiovascular magnetic resonance physics for clinicians: Part II. J Cardiovasc Magn Reson. 2012;14:66.

13. Peters DC, Appelbaum EA, Nezafat R, Dokhan B, Han Y, Kissinger KV, et al. Left ventricular infarct size, peri-infarct zone, and papillary scar measurements: A comparison of high-resolution $3 \mathrm{D}$ and conventional $2 \mathrm{D}$ late gadolinium enhancement cardiac MR. J Magn Reson Imaging. 2009;30:794-800.

14. O'Brien KR, Gabriel RS, Greiser A, Cowan BR, Young AA, Kerr AJ. Aortic valve stenotic area calculation from phase contrast cardiovascular magnetic resonance: the importance of short echo time. J Cardiovasc Magn Reson. 2009;11:49.

15. O'Brien KR, Cowan BR, Jain M, Stewart RA, Kerr AJ, Young AA. MRI phase contrast velocity and flow errors in turbulent stenotic jets. J Magn Reson Imaging. 2008;28:210-8.

16. Stahlberg F, Sondergaard L, Thomsen C, Henriksen O. Quantification of complex flow using MR phase imaging-a study of parameters influencing the phase/velocity relation. Magn Reson Imaging. 1992;10:13-23.

\section{Submit your next manuscript to BioMed Central and take full advantage of:}

- Convenient online submission

- Thorough peer review

- No space constraints or color figure charges

- Immediate publication on acceptance

- Inclusion in PubMed, CAS, Scopus and Google Scholar

- Research which is freely available for redistribution

Submit your manuscript at www.biomedcentral.com/submit
C) Biomed Central 